\title{
FAIRNESS INDICES AND DISTRIBUTED CONTROL IN COMMUNICATION NETWORKS
}

\author{
WING SHING WONG* AND FENGJUN LI*
}

\begin{abstract}
The concept of fairness index for self-authority servers in a large-scale network is introduced in this paper. The index quantifies the relative contributions of the servers to network routing, and can be used in network administration processes, such as negotiation of Multi-Lateral Peering Agreements. The fairness index concept leads naturally to the idea of an absolutely fair solution, which is a study focus in this paper. Although, an absolutely fair solution may not be an ideal operating point due to efficiency considerations, it serves as a reference point for comparing contribution from various servers in a network. Uniqueness and existence properties of absolutely fair solutions are examined in general as well as for certain specially structured networks of interest. Via the concept of a pricing duality, the connection of absolutely fair solutions to the von Neumann economic model is established. For implementation considerations, a distributed, low-data-rate control algorithm that converges to pre-defined fairness index targets is introduced and analyzed. A heuristic extension is studied to provide a practical approach for realistic situations.
\end{abstract}

Keywords: Fairness Index, von Neumann Equilibrium, Distributed low data rate controller.

1. Introduction. Open architecture networks, such as the Internet, are built on the premise that all network nodes participate in the routing of third party traffic whenever demand is under resource capacity. This altruistic spirit helped the Internet grow to its current level of development in a relatively short time. However, if one analyzes the Internet architecture a bit deeper, one finds that it is not as altruistic as one may imagine. For example, a backbone server typically only connects to other backbone network servers of similar caliber [1]. A Bi-Lateral Peering Agreement (BLPA) between two parties is signed only if the two traffic flows coming from each party to the other are comparable in size. End users are not granted direct connectivity; instead, connectivity is achieved through network access servers, provided, for example, by an Internet Service Provider. This kind of arrangement can be interpreted as a scheme to ensure fairness in routing contribution: similarly sized backbone servers make roughly the same amount of routing contribution to each other, while smaller servers must aggregate their traffic through backbone servers, typically for a price. Since limiting peering agreements to the bilateral type only is restrictive, the so-called Multi-Lateral Peering Agreement (MLPA) has been proposed. However, the fairness issue in a multi-lateral set up is much more complicated than in bi-lateral cases as servers can form a "fair" peering group without satisfying pair-wise symmetric traffic

${ }^{*}$ Department of Information Engineering, Chinese University of Hong Kong. E-mail: wswong @ie.cuhk.edu.hk, fli@ist.psu.edu. The work reported in this paper was partially supported by a Direct Grant of the Chinese University of Hong Kong. 
conditions.

The fairness issue is even more critical for a wireless ad-hoc network. Unlike hierarchical networks, these networks are self-organizing with all nodes acting as their own authorities. Unlike servers in a wired network, mobile servers are highly constrained by battery power resources. Routing for third party traffic consumes not only computation and channel bandwidth but battery power as well. Hence, fairness in routing becomes an even more critical issue in such networks.

In this paper, a concept called fairness index is introduced. An early version of this concept was first proposed in [2] and later in [3]. This index is defined for each node and can be evaluated by each node independently. It is an indicator of the routing contribution of a node to the network. The ideal case where all the nodes have identical fairness indices is an important baseline reference point. The corresponding network is known as absolutely fair, (this replaces the terminology of perfectly fair used in [2] and [3].) It would be too stringent to require that networks operate at such an ideal, absolutely fair state. However, knowledge of how a network deviates from the ideal state can provide valuable information and help one decide whether peering agreements among server nodes are justifiable.

One can generalize the concept of an absolutely fair solution to a von Neumann equilibrium solution by introducing a duality concept of pricing in the network. A von Neumann equilibrium solution always exists under very general conditions.

For practical considerations, one needs to consider the problem of how a network can achieve an absolutely fair state if that is indeed the network goal. Since each node can only have a local, partial observation of the network, the control to achieve the desired performance goal should be preferably achieved through a distributed algorithm. This problem is further complicated by the fact that network parameters are typically unknown. Nevertheless, in this paper a simple, distributed controller will be presented and shown to converge to any feasible fairness index target, under suitable technical conditions. The algorithm can be extended to a heuristic approach for convergence to an absolutely fair state even when the fairness index is not known a priori.

The organization of the paper is as follows. In section 2, the basic network considered in this paper is defined and concepts of fairness index and absolutely fair solutions are introduced. In section 3, existence and uniqueness properties of an absolutely fair solution are examined. In section 4, connection with the von Neumann equilibrium is explained. In section 5 , issues of using a distributed controller to achieve fairness index targets are discussed. A main result is to establish the convergence of a class of distributed, low data rate feedback controllers. In section 6 , numerical examples are provided to illustrate the practicality of the distributed controller. A 
heuristic algorithm for convergence to an absolutely fair state is also discussed. Section 7 provides some concluding remarks.

2. Concept of Fairness Index. Conceptualize a communication network as a graph $(V, E)$, with nodes 1 to $K(K \geq 2)$ representing servers; an edge connects two nodes whenever there is a direct (full duplex) data link between two servers. A node in the network $(V, E)$ receives traffic from other nodes, sends traffic to other nodes, and routes traffic that can originate from and destine to anywhere in the network.

A basic hypothesis here is that each node is an independent administrative entity and it acts to maximize its own interest under pre-defined rules subjected to capacity constraints of both links and nodes. To measure whether a peering relationship is equitable, one can define a fairness index for each node, which can be understood to be the ratio of traffic directly attributed to a node as either a source or a destination to the total traffic it handles.

For each node $j$, represent by $r_{j}$ the total traffic generated by node $j$ to the network. Denote the vector of network input traffic rates, $\left(r_{1}, \cdots, r_{K}\right)^{\mathrm{T}}$, by $\mathbf{r}$. The nodes control the network by adjusting the vector $\mathbf{r}$.

For $i \neq j$, let $M_{i j} r_{j}$ represent the rate of the input traffic from node $j$ that is ultimately destined for node $i$. For all $i$, define $M_{i i}=0$. Denote by $\mathbf{M}$ the following $K$ by- $K$ input traffic distribution matrix, which is a non-negative matrix with constant column sums equal to 1 ,

$$
\left(\begin{array}{llll}
0 & M_{12} & \cdots & M_{1 K} \\
M_{21} & 0 & \cdots & M_{2 K} \\
\vdots & \vdots & \ddots & \vdots \\
M_{K 1} & M_{K 2} & \cdots & 0
\end{array}\right)
$$

Traffic from any source-destination pair can be routed over a variety of paths. It is assumed that for any source-destination pair, the distributions of traffic into these alternative paths are arbitrary but known and remain unchanged in a sufficiently long enough time period for the consideration of this problem.

Since data traffic can travel through intermediate nodes, given an input traffic distribution matrix and a set of fixed routing schemes, the distribution of transitory traffic in the networks is accordingly fixed. Let $L_{i j} r_{j}$ denote the traffic rate of transitory traffic passing through node $i$ that originates from node $j$, and $\mathbf{L}=\left(L_{i j}\right)$ denote the corresponding transitory traffic distribution matrix. $\mathbf{L}$ is also a non-negative matrix with all its entries are bounded by $1, L_{i i}=0$, and $\mathbf{L r}$ is the column vector representing the total transitory data traffic passing through each node. Note that the traffic flow represented by the sum $\sum_{j=1}^{K} L_{i j} r_{j}$ is distinct from the traffic flow represented by $\sum_{j=1}^{K} M_{i j} r_{j}$. As an example, consider a network when all traffic are 
routed directly from source node to destination node, in this case, $\sum_{j=1}^{K} L_{i j} r_{j}=0$ but $\sum_{j=1}^{K} M_{i j} r_{j}$ cannot vanish for all $i$ unless all the $r_{i}$ 's are zero.

In order to control selfish behaviors in a network, behaviors that may cause serious network congestion, many investigations focus on utility optimization by using pricing scheme and game theory. By means of the max-min fairness defined in [4], the proportional fairness concept [5-7] and its generalization [8], or the Nash equilibrium as in [9], various fairness concepts for network flow control have been investigated. However, these investigations did not consider the fairness issue from the viewpoint of the peering nodes. The proposed fairness index in this paper measures fairness by calculating the normalized routing contribution to the network by an individual node. Depending on the economical model one adopts to account for the utility of the traffic, there are three classes of fairness indices.

Definition 1. For a network, $(V, E)$, if $r_{i}+\sum_{j=1}^{K}\left(M_{i j}+L_{i j}\right) r_{j}>0$, the sourcefairness index, $\iota_{i}$, the destination-fairness index, $o_{i}$, and the source-destinationfairness index, $\chi_{i}$, for node $i$ are defined as follows.

$$
\begin{aligned}
\iota_{i} & =\frac{r_{i}}{r_{i}+\sum_{j=1}^{K}\left(M_{i j}+L_{i j}\right) r_{j}}, \quad o_{i}=\frac{\sum_{j=1}^{K} M_{i j} r_{j}}{r_{i}+\sum_{j=1}^{K}\left(M_{i j}+L_{i j}\right) r_{j}}, \\
\text { and } \chi_{i} & =\frac{r_{i}+\sum_{j=1}^{K} M_{i j} r_{j}}{r_{i}+\sum_{j=1}^{K}\left(M_{i j}+L_{i j}\right) r_{j}} .
\end{aligned}
$$

Otherwise all the indices are defined to be zero.

Note that all these indices take values between 0 and 1 and that the denominators of all these indices are identical and account for the total traffic handled by node $i$. For the source-fairness index, the numerator accounts for the total data rate originated from a node to the network. Traffic received by a node as final destination is assumed to have no economical benefit to it. For the destination-fairness index, the numerator accounts for traffic received by a node as final destination. Traffic originating from a node is assumed to have no economical benefit to it in this case. For the case where originating and receiving destination traffics are both considered to possess economical value to a node, one should use the source-destination-fairness index instead.

To fix concept for subsequent discussion, note that a vector $\mathbf{x}$ is positive if it is non-zero and all its components are non-negative. The notation $\mathbf{x}>0$ is used. $\mathbf{x}$ is strictly positive if all its components are strictly positive and the notation $\mathbf{x} \gg 0$ is used. One can extend the same convention for matrices obviously.

DEFINITION 2. An absolutely source-destination-fair solution exists if there is a 
positive rate vector, $\mathbf{r}=\left(r_{1}, \cdots, r_{K}\right)^{\mathrm{T}}$, so that the source-destination-fairness indices for all nodes are equal.

One can define absolutely source-fair or absolutely destination-fair solutions in a similar way. To make the nomenclature less cumbersome, an absolutely sourcedestination-fair solution will be described as simply source-destination-fair. Sourcefair or destination-fair solutions will be used to describe absolutely source-fair or absolutely destination-fair solutions respectively. It is easy to show that if a sourcedestination-fair solution exists, the corresponding index must be strictly positive. Moreover, a source-fair solution exists if and only if there is a $\gamma_{S}>0$ so that $\iota_{i}=\gamma_{S}$ for all $0 \leq i \leq K$, and a strictly positive rate vector $\mathbf{r}$ exists so that

$$
\gamma_{S}(\mathbf{I}+\mathbf{L}+\mathbf{M}) \mathbf{r}=\mathbf{r},
$$

where I stands for the $K$-by- $K$ identity matrix, and $\gamma_{S}$ will be called the sourcefairness index of the pair $(\mathbf{M}, \mathbf{L})$. Similarly a destination-fair solution (respectively source-destination-fair solution) exists if and only if there exists a $\gamma_{D}>0$ (respectively a $\gamma_{C}>0$ ) and a positive rate vector $\mathbf{r}$ so that equation (2.4) (respectively (2.5)) is satisfied.

$$
\begin{gathered}
\gamma_{D}(\mathbf{I}+\mathbf{L}+\mathbf{M}) \mathbf{r}=\mathbf{M r} . \\
\gamma_{C}(\mathbf{I}+\mathbf{L}+\mathbf{M}) \mathbf{r}=(\mathbf{I}+\mathbf{M}) \mathbf{r} .
\end{gathered}
$$

$\gamma_{D}$ and $\gamma_{C}$ are known as the destination-fairness index and source-destination-fairness index of the pair $(\mathbf{M}, \mathbf{L})$ respectively. The existence and uniqueness properties of various fair solutions are natural questions for investigation. For source-fair solutions, the question can be settled by using the Perron-Frobenius Theorem on non-negative matrices [10]. The other two cases are much more complicated and will be investigated in section 3. The maximal $\gamma_{C}$ satisfying equation (2.5) is called the source-destinationfairness index for the network. The other two fairness indices can be defined similarly.

Note that fair solutions can be scaled uniformly without affecting their fairness properties. Hence, if any fair solution exists, another one can always be found that satisfies a given set of link and node capacity constraints. Such a solution, of course, may not utilize the network efficiently, as there is usually a trade-off between efficiency versus fairness. Using the conventionally defined concept of network utilization, a fair solution may not be the best operating point. However, for other discussion on the tradeoff one can read [11]. If a fairness solution does not exist, then one may need to consider a different set of routing schemes, and hence a different transitory traffic distribution matrix $\mathbf{L}$. 
3. Basic Existence and Uniqueness Properties. In this section, existence and uniqueness properties of the various fair indices are investigated.

Lemma 1. The value of any of the three defined types of absolutely fair indices is positive and less than or equal to 1 .

Proof of this statement is straightforward and is omitted here.

Proposition 1. For any $\mathbf{M}$ described in (2.1) and any non-negative $\mathbf{L}$, an absolutely source-fair index solution always exists. If $\mathbf{I}+\mathbf{L}+\mathbf{M}$ is irreducible, then the index, $\gamma_{S}$, is uniquely defined and the corresponding rate vector is strictly positive and unique up to a scalar constant.

Proof. Suppose $\mathbf{I}+\mathbf{L}+\mathbf{M}$ is irreducible. The existence and uniqueness claim of the theorem follows directly from the Perron-Frobenius Theorem on irreducible matrices (see for example [10].) If $\mathbf{I}+\mathbf{L}+\mathbf{M}$ is not irreducible, then there is a permutation matrix $\mathbf{P}$ so that $\mathbf{P}(\mathbf{I}+\mathbf{L}+\mathbf{M}) \mathbf{P}^{\mathrm{T}}=\mathbf{I}+\mathbf{P}(\mathbf{L}+\mathbf{M}) \mathbf{P}^{\mathrm{T}}$ is in upper block-triangular form with all diagonal terms equal to 1 . The topmost block is again in the form of an identity matrix (of a smaller dimension) plus a non-negative matrix. Moreover, it is clear that if that block sub-matrix has an eigenvector then it can be extended to an eigenvector for $\mathbf{I}+\mathbf{L}+\mathbf{M}$. So by induction arguments, one can show that $\mathbf{I}+\mathbf{L}+\mathbf{M}$ also has a positive eigenvector with strictly positive eigenvalue. One can conclude that the existence part of the theorem always holds.

The uniqueness and existence issue for the other two types of indices are much more complicated. Mathematically, the issue hinges on finding generalized positive eigenvectors for a pair of nonnegative matrices. Very few results have been reported in the literature on this subject, one approach to the problem is provided by Mangasarian [9]. In the following, results related to this approach are presented. For any $K$ by- $K$ matrix, $\mathbf{R}=\left[\mathbf{R}_{1}, \ldots \mathbf{R}_{K}\right]$, define the cone, $C(\mathbf{R})$, to be the set of the form $\left\{\sum_{i} c_{i} \mathbf{R}_{i}: c_{i} \geq 0\right\}$. An interior point of $C(\mathbf{R})$ is a vector of the form $\sum_{i} c_{i} \mathbf{R}_{i}$ with all $c_{i}>0$.

Proposition 2.

1. Suppose $C(\mathbf{I}+\mathbf{L}) \subseteq C(\mathbf{M})$, then a destination-fair solution, $\left(\gamma_{D}, \mathbf{r}\right)$, to $(2.4)$ exists with $0<\gamma_{D}<1$.

2. If $C(\mathbf{I}+\mathbf{L})$ is contained in the interior of $C(\mathbf{M})$ and $\mathbf{M}$ is invertible, then $\gamma_{D}$ is uniquely defined, $\mathbf{r}$ is strictly positive and unique up to a scalar constant.

Proof. For statement 1, note that $C(\mathbf{I}+\mathbf{L}) \subseteq C(\mathbf{M})$ implies any column of $\mathbf{I}+\mathbf{L}$ is contained in the cone $C(\mathbf{M})$. Hence, there is $(\mathbf{I}+\mathbf{L})=\mathbf{M C}$ for some positive matrix C. It follows that the spectral radius of $\mathbf{C}, \rho(\mathbf{C})$, is an eigenvalue with a nonnegative eigenvector, $\mathbf{r}$, (see for example [10]). Then

$$
(\mathbf{I}+\mathbf{L}) \mathbf{r}=\mathbf{M C r}=\rho(\mathbf{C}) \mathbf{M r} .
$$


It follows that $\rho(\mathbf{C}) \neq 0$ since $(\mathbf{I}+\mathbf{L}) \mathbf{r} \neq 0$. Moreover,

$$
(\mathbf{I}+\mathbf{M}+\mathbf{L}) \mathbf{r}=\mathbf{M C r}=(1+\rho(\mathbf{C})) \mathbf{M r} .
$$

Hence, an absolutely destination-fair solution to (2.4) exists with $\gamma_{D}=1 /(1+$ $\rho(\mathbf{C})), 0<\gamma_{D}<1$.

For the second statement, note that if $C(\mathbf{I}+\mathbf{L})$ is contained in the interior of $C(\mathbf{M})$, then the matrix $\mathbf{C}$ defined earlier is a strictly positive matrix. Hence, by the Perron-Frobenius Theorem $\rho(\mathbf{C})$ is the unique eigenvalue of $\mathbf{C}$ with a strictly positive eigenvector, $\mathbf{r}$. Then any absolutely destination-fair solution, $\mathbf{r}^{\prime}$ with fairness index $\gamma^{\prime}$ satisfies

$$
\begin{aligned}
& \gamma^{\prime}(\mathbf{I}+\mathbf{L}+\mathbf{M}) \mathbf{r}^{\prime}=\mathbf{M r}^{\prime}=\gamma^{\prime}(\mathbf{M C}+\mathbf{M}) \mathbf{r}^{\prime} \\
& \left(1 / \gamma^{\prime}-1\right) \mathbf{M r} \mathbf{r}^{\prime}=\mathbf{M C \mathbf { r } ^ { \prime }} .
\end{aligned}
$$

If $\mathbf{M}$ is invertible, then this implies $1 / \gamma^{\prime}-1$ is an eigenvalue of $\mathbf{C}$ with strictly positive eigenvector. Hence $1 / \gamma^{\prime}-1=\rho(\mathbf{C})$ and $\mathbf{r}^{\prime}$ is equal to $\mathbf{r}$ up to a scalar multiple.

Similarly, one can establish the following result:

PROPOSITION 3.

1. Suppose $C(\mathbf{I}+\mathbf{M}) \subseteq C(\mathbf{L})$, then a source-destination-fair solution, $\left(\gamma_{C}, \mathbf{r}\right)$, to (2.5) exists.

2. If $C(\mathbf{I}+\mathbf{M})$ is contained in the interior of $C(\mathbf{L})$ and $\mathbf{L}$ is of full rank, then $\gamma_{C}$ is uniquely defined, $\mathbf{r}$ is strictly positive and unique up to a scalar constant.

For networks with a special architecture, it is possible to obtain more specific characterizations. For illustration, consider two classes of networks, which represent two extreme scenarios: uniform routing network and single routing node network.

DEFINITION 3. A network has uniform routing if it is a fully connected network of $K$ nodes and any traffic between two distinct nodes is routed through any one of the remaining $K-2$ nodes with a predefined proportion $c, 0<c<1$.

Let

$$
\mathbf{K} \equiv\left[\begin{array}{llll}
\mathbf{e} & \mathbf{e} & \cdots & \mathbf{e}
\end{array}\right] \equiv\left[\begin{array}{cccc}
1 & 1 & \cdots & 1 \\
1 & 1 & \cdots & 1 \\
\vdots & \vdots & \ddots & \vdots \\
1 & 1 & \cdots & 1
\end{array}\right]
$$

The transitory traffic matrix, $\mathbf{L}$, for a uniform routing network is given by:

$$
\mathbf{L}=c((\mathbf{K}-\mathbf{I}) \mathbf{M}-\mathbf{I})=c(\mathbf{K M}-\mathbf{M}-\mathbf{I}) .
$$

One way to check that this is correct is to note that the traffic flow from node $j$ to $i$ with rate $M_{i j} r_{j}$ would have a transitory traffic contribution at rate $c M_{i j} r_{j}$ according 
to (3.5) to any node that is distinct from $i$ and $j$ and zero to nodes $i$ and $j$. Since any columns of $\mathbf{M}$ sum to one, it is easy to see that $\mathbf{K M}=\mathbf{K}$ and

$$
\mathbf{L}=c(\mathbf{K}-\mathbf{M}-\mathbf{I}) .
$$

Equation (2.5) and equation (2.4) can be re-written as

$$
\left(\frac{1}{\gamma_{C}}-1+c\right)(\mathbf{I}+\mathbf{M}) \mathbf{r}=c \mathbf{K r},
$$

and

$$
\frac{1}{\gamma_{D}} \mathbf{M r}+(c-1)(\mathbf{I}+\mathbf{M}) \mathbf{r}=c \mathbf{K r} .
$$

For any real $s$, define $\mathbf{N}_{s}=\mathbf{M}-s \mathbf{K}$.

Proposition 4. For a uniform routing network, the following holds.

1. If (3.7) has a positive solution, then $\gamma_{C}=2 /(c K+2(1-c))$.

2. Assume that $\mathbf{M}$ is primitive and $\max _{i, j} \mathbf{M}_{i j}=s<\frac{2}{K}$. Then, the vector

$$
\mathbf{r}=\left(\mathbf{I}+\mathbf{N}_{s}\right)^{-1} \mathbf{e}=\left(\mathbf{I}-\mathbf{N}_{s}+\mathbf{N}_{s}^{2}-\cdots\right) \mathbf{e}
$$

is a strictly positive solution to (3.7) and unique up to a positive scalar constant.

Proposition 5. For a uniform routing network, the following holds.

1. If (3.8) has a positive solution, then $\gamma_{D}=1 /(c K+2(1-c))$.

2. If $\min _{i, j} \mathbf{M}_{i j}>\frac{c}{1+c(K-1)}$, then (3.8) has a strictly positive solution which is unique up to multiplication by a positive scalar.

Details of the proofs of the above two propositions can be found in the appendix. Hence for uniformly routing networks, absolutely source-destination-fair and absolutely destination-fair solutions exist under suitable conditions. This is not the case for single routing node networks.

DEFINITION 4. A single routing node network is a network such that traffic between any source-destination pair is delivered either directly or routed through a unique node.

For such a network, by re-labeling the nodes if necessary, one can represent $\mathbf{M}$ and $\mathbf{L}$ in the following form:

$$
M=\left[\begin{array}{ll}
0 & M_{12}^{\mathrm{T}} \\
M_{21} & M_{22}
\end{array}\right],
$$

and

$$
L=\left[\begin{array}{llll}
0 & L_{12} & \cdots & L_{1 K} \\
0 & 0 & \cdots & 0 \\
\vdots & \vdots & \ddots & \vdots \\
0 & 0 & \cdots & 0
\end{array}\right]=\left[\begin{array}{ll}
0 & L_{12}^{\mathrm{T}} \\
0_{K-1,1} & 0_{K-1, K-1}
\end{array}\right]
$$


As stated previously, the source-fair index always exists. For the source-destinationfair index, one can show by inspection that the corresponding equation

$$
\mathbf{L r}=\frac{1-\gamma_{C}}{\gamma_{C}}(\mathbf{I}+\mathbf{M}) \mathbf{r}
$$

has only a solution with $\gamma_{C}=1, r_{1}>0, r_{2}=\cdots r_{K}=0$. However, this implies a decoupled network as there is no non-directly routed traffic. In other words, the source-destination-fair index cannot be meaningfully defined for this class of networks. The situation is completely different for destination-fair solutions.

Proposition 6. A single routing node network has a destination-fair solution if

$$
\left(M_{12}-\rho\left(M_{22}\right) L_{12}^{\mathrm{T}}\right) z>0
$$

where $\rho\left(M_{22}\right)$ is the spectral radius of $M_{22}$ and $z$ is a corresponding eigenvector.

The proof of the above proposition can be found in the appendix.

For an illustration of Proposition 6 and the meaning of (3.13), consider a threenode single routing network as in Figure 1.

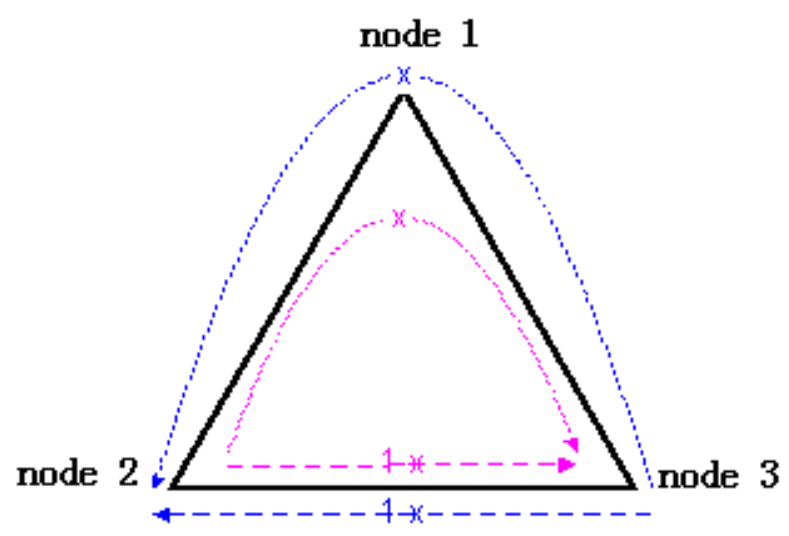

Figure 1

The routing scheme is specified as follows. Node 1 is the single routing node, which sends traffic to the other nodes directly. Traffic between node 2 and node 3 are either sent directly or pass through node 1 as a transitory node. The proportions of routed traffic through node 1 originated from node 2 and node 3 are assumed to be the same and represented by $x, 0 \leq x \leq 1$. For illustration, we pick a special realization of the matrix $\mathbf{M}$ and parameterize the transitory traffic distribution matrix $\mathbf{L}$ by $x$ :

$$
M=\left[\begin{array}{ccc}
0 & 0.19 & 0.36 \\
0.50 & 0 & 0.64 \\
0.50 & 0.81 & 0
\end{array}\right] \text {, and } L=\left[\begin{array}{ccc}
0 & 0.81 x & 0.64 x \\
0 & 0 & 0 \\
0 & 0 & 0
\end{array}\right] \text {. }
$$


To study absolutely destination-fair solution of this example network, numerical computations were carried out to track the destination-fairness index as $x$ varies from 0 to 1 as shown in the following figures.

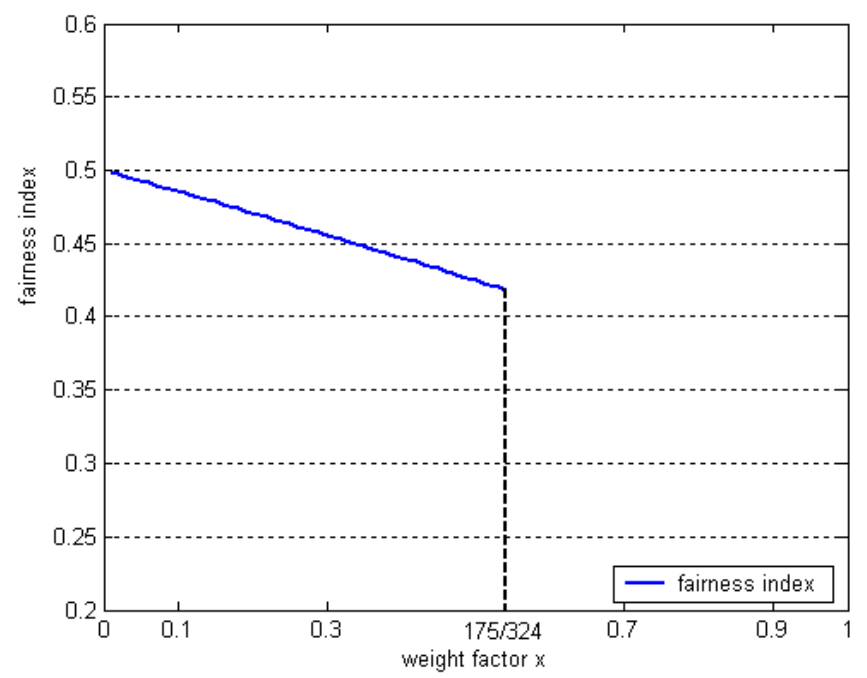

Figure 2

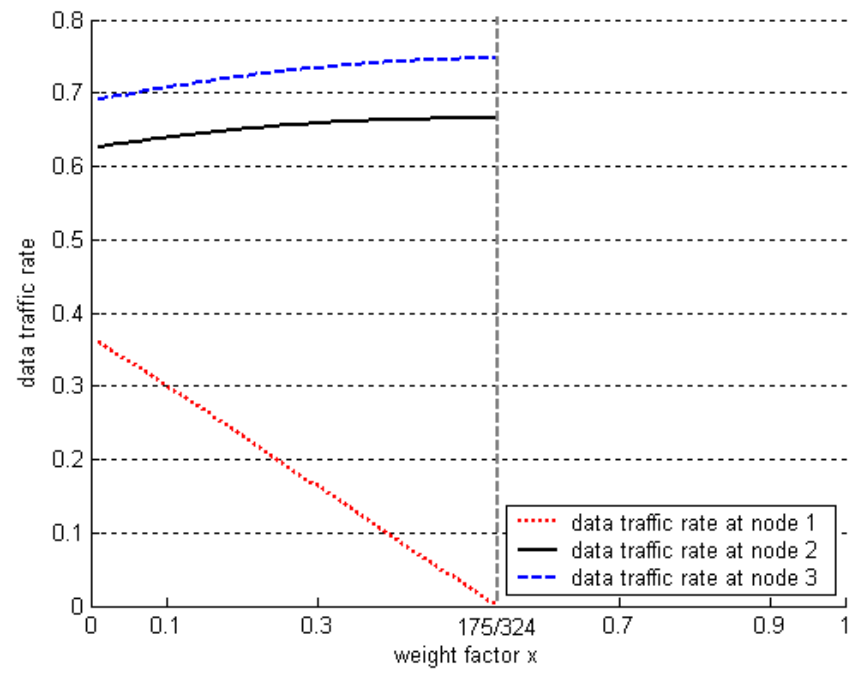

Figure 3

From Figure 2, one can see that the maximum fairness index value for these examples is achieved at the point $x=0$, in which case data traffic of the whole network are all routed directly. As the routed traffic increases, the index decreases 
monotonically until reaching the point $x=175 / 324$. Beyond this value, the fairness index does not exist for such a class of systems.

A way to gain more insight about this is to examine the corresponding traffic rates of the nodes as a function of $x$. Trajectories for the three data traffic rates are shown in the Figure 3. One can see the data traffic of the routing node decreases monotonically while the traffic rates at the other two nodes increase monotonically. This change is due to the need to compensate for the decrease of the fairness index at the routing node caused by the increase of transitory traffic passing through it. At the point $x=175 / 324$, the traffic rate of node 1 drops to 0 . So intuitively speaking, beyond the point, no further adjustment is feasible to maintain an absolutely balanced solution.

For a more theoretical analysis, one can check the sufficient condition in Proposition 6 for the existence of a destination-fair solution for such a network. Rewrite the input traffic distribution matrix $\mathbf{M}$ and the corresponding transitory traffic matrix $\mathbf{L}$ as:

$$
\begin{aligned}
M & =\left[\begin{array}{lll}
0 & 1-b & 1-a \\
c & 0 & a \\
1-c & b & 0
\end{array}\right]=\left[\begin{array}{ll}
0 & M_{12}^{\mathrm{T}} \\
M_{21} & M_{22}
\end{array}\right], \\
\text { and } L & =\left[\begin{array}{lll}
0 & b x & a x \\
0 & 0 & 0 \\
0 & 0 & 0
\end{array}\right]=\left[\begin{array}{ll}
0 & L_{12}^{\mathrm{T}} \\
0_{K-1,1} & 0_{K-1, K-1}
\end{array}\right] .
\end{aligned}
$$

Then the dominant positive eigenvalue of $M_{22}$ is $\sqrt{a b}$ and a corresponding eigenvector is $[\sqrt{a}, \sqrt{b}]^{\mathrm{T}}$. Equation (3.11) turns to be equivalent to

$$
\left(M_{12}^{\mathrm{T}}-\rho\left(M_{22}\right) L_{12}^{\mathrm{T}}\right) z=\left[\begin{array}{ll}
1-b-\sqrt{a b} x & 1-a-\sqrt{a b} x
\end{array}\right]\left[\begin{array}{c}
\sqrt{a} \\
\sqrt{b}
\end{array}\right]>0 .
$$

For the network parameters used in the example, this expression can be simplified as

$$
x<\frac{1-\sqrt{a b}}{a b}=\frac{175}{324} .
$$

The numerical result matches the analytical result well. The example shows that under certain circumstances there may not be any absolutely fair solution. In this case a different set of routings need to be adopted. If there is no absolutely fair solution for all routing arrangements, this may imply that no peering agreement should exist for the network. When there is no absolutely fair solution, an alternative is to bring in a concept of pricing. This latter approach makes contact with an equilibrium concept proposed by von Neumann [14]. 
4. Von Neumann Equilibrium. To extend the absolutely fair concept to a more general context, in particular for those cases where the fairness index may not exist, one approach is to use the fairness index to define a minimum level of contribution to the network. More specifically, instead of requiring all the fairness indices are equal, one could set a requirement that the indices should be above certain target level. Consequently one is interested in finding input traffic rates that ensure the minimum fairness index among all nodes is maximized. To illustrate the concept more concretely, let $\theta_{i}(\mathbf{r})$ represent the source-destination-fairness index of node $i$ when the rate vector is $\mathbf{r}$. One can define a network index,

$$
\sigma_{C}=\sup _{\mathbf{r}} \min _{i} \theta_{i}(\mathbf{r})
$$

where the supremum is taken over all positive vectors, $\mathbf{r}$. Since $\theta_{i}(\mathbf{r})$ is homogenous in $\mathbf{r}$, (that is $\theta_{i}(c \mathbf{r})=\theta_{i}(\mathbf{r})$ for any $c>0$ ), it can be regarded as a function defined on a compact set and hence the supremum is achieved by a maximum. So that

$$
\sigma_{C}=\max _{\mathbf{r}} \min _{i} \theta_{i}(\mathbf{r})
$$

Moreover,

$$
1 / \sigma_{C}=\min _{\mathbf{r}} \max _{i} 1 / \theta_{i}(\mathbf{r}) .
$$

Define $\sigma_{S}$ and $\sigma_{D}$ similarly using source-fairness and destination-fairness indices respectively.

If a source-destination-fairness index of the pair ( $\mathbf{M}, \mathbf{L})$ exists, then the corresponding rate vector defining the index is among the r's in the right-hand-side of equation (4.2), hence

$$
\gamma_{C} \leq \sigma_{C}
$$

One can show similarly that

$$
\begin{gathered}
\gamma_{S} \leq \sigma_{S}, \\
\gamma_{D} \leq \sigma_{D} .
\end{gathered}
$$

In fact, if $\mathbf{M}+\mathbf{L}+\mathbf{I}$ is irreducible, then by the Perron-Frobenius Theorem again, it follows that the inequality in (4.5) is an equality.

Another approach to extend the absolutely fair concept is to introduce a positive shadow price vector, $p=\left(p_{1}, \ldots, p_{K}\right)$, as a "dual" vector to the traffic rate vector. The term $p_{i}$ represents the price per unit traffic charged by node $i$ for all data traffic handled by it. That is, node $i$ always charges at $p_{i}$ dollars per unit traffic no matter 
whether it is for the traffic it generates, traffic it receives, or traffic it transfers as an intermediate node.

Consider now one unit of data traffic generated by the node $j$. As the traffic routes through the network, the amount of fee charged by various nodes in the network is given by

$$
p_{j}+\sum_{i=1}^{K} p_{i}\left(M_{i j}+L_{i j}\right) .
$$

Note that the fees are paid to different nodes. For example, $p_{j}$ is paid to node $j$ for generating the traffic and the amount $p_{i}\left(M_{i j}+L_{i j}\right)$ is paid to node $i \neq j$, where $p_{i} M_{i j}$ is for receiving the traffic that goes to node $i$ and $p_{i} L_{i j}$ for transferring the traffic that goes to other nodes.

Suppose that there are economic values for the end-to-end traffic carried on the network so that both source and destination nodes can benefit at the rate of $\Upsilon p_{i}$ per unit traffic generated or received, where $\Upsilon$ is a network-wide constant. Then, the economic value of the one unit of traffic rate generated by node $j$ is given by

$$
\Upsilon\left[p_{j}+\sum_{i=1}^{K} p_{i} M_{i j}\right] .
$$

Note that the economic values are collected by various node in the network for different reasons. For example $p_{j} \Upsilon$ represents the fee collected by node $j$ for generating that one unit of traffic, while $\Upsilon p_{i} M_{i j}$ represents the fee collected by node $i,(i \neq j$,$) for$ receiving the traffic that goes to it. Note that there is no economic value of transitory traffic.

To measure the economic efficiency of various data traffic streams, one can define a source-destination-pricing efficiency index as

$$
\chi_{j}^{p}=\Upsilon \frac{p_{j}+\sum_{i=1}^{K} p_{i} M_{i j}}{p_{j}+\sum_{i=1}^{K} p_{i}\left(M_{i j}+L_{i j}\right)},
$$

If $p_{j}+\sum_{i=1}^{K} p_{i}\left(M_{i j}+L_{i j}\right)>0$. Since $\Upsilon$ is a system-wide constant, one can normalize it to one and reduces (4.9) to

$$
\chi_{j}^{p}=\frac{p_{j}+\sum_{i=1}^{K} M_{i j} p_{i}}{p_{j}+\sum_{i=1}^{K}\left(M_{i j}+L_{i j}\right) p_{i}} .
$$

Similarly one can define the source-pricing index and the destination-pricing index if the economic values are restricted to the source or destination respectively only. 
Definition 5. For a network, $(V, E)$, if $p_{j}+\sum_{i=1}^{K} p_{i}\left(M_{i j}+L_{i j}\right)>0$, the sourcepricing efficiency index, $\iota_{j}^{p}$, the destination-pricing efficiency index, $o_{j}^{p}$ and the sourcedestination-pricing efficiency index, $\chi_{j}^{p}$, for the data stream generated at node $j$ are defined as follows.

$$
\begin{aligned}
\iota_{j}^{p} & =\frac{p_{j}}{p_{j}+\sum_{i=1}^{K}\left(M_{i j}+L_{i j}\right) p_{i}}, \quad o_{j}^{p}=\frac{\sum_{i=1}^{K} M_{i j} p_{i}}{p_{j}+\sum_{i=1}^{K}\left(M_{i j}+L_{i j}\right) p_{i}}, \\
\chi_{j}^{p} & =\frac{p_{j}+\sum_{i=1}^{K} M_{i j} p_{i}}{p_{j}+\sum_{i=1}^{K}\left(M_{i j}+L_{i j}\right) p_{i}} .
\end{aligned}
$$

Otherwise the indices are defined to be zero.

By varying the shadow prices, one can obtain different pricing efficiency indices for the network nodes. Pricing schemes that lead to high overall pricing efficiencies may be regarded as more desirable, hence providing a way to determine the shadow prices. Note that all these pricing indices take values between 0 and 1 similar to the fairness indices.

With pricing, one can extend the absolutely fair concept by means of the von Neumann economic model [14]. In the current context, it is defined via a set of $2 K$ equations, taking one of the following forms depending which type of indices is used:

$$
\left\{\begin{array}{l}
\alpha_{C} \mathbf{p}(\mathbf{I}+\mathbf{M}) \leq \mathbf{p}(\mathbf{I}+\mathbf{L}+\mathbf{M}), \quad \text { for source - destination - based indices. } \\
\beta_{C}(\mathbf{I}+\mathbf{M}) \mathbf{r} \geq(\mathbf{I}+\mathbf{L}+\mathbf{M}) \mathbf{r} .
\end{array}\right.
$$

In order to facilitate subsequent discussions, it is more convenient to make the following notation change. Use $\mathbf{A}$ and $\mathbf{B}$ to denote respectively the $K$-by- $K$ nonnegative matrices in the left hand side and right hand side of one of the equations in (4.12), (4.13) or (4.14). These equations can be re-written in a general form

$$
\left\{\begin{array}{l}
\alpha \mathbf{p A} \leq \mathbf{p B} \\
\beta \mathbf{A r} \geq \mathbf{B r}
\end{array}\right.
$$

For all three types of indices, matrix $\mathbf{B}$, taking the same form as $(\mathbf{I}+\mathbf{L}+\mathbf{M})$ and represents the total traffic distribution matrix that accounts for all source traffic, 
destination traffic, and transitory traffic. While matrix $\mathbf{A}$, is a traffic distribution matrix that depends on which type of traffic is considered valuable. For ease of discussion, the source-destination-fairness model is assumed below. It is straightforward to extend the discussion to the other two types of models.

From a viewpoint of fairness, each node, $i$, sends and receives data traffic of interest to it amounting to $\sum_{j=1}^{K} a_{i j} r_{j}=r_{i}+\sum_{j=1}^{K} M_{i j} r_{j}$, while its total contribution to the network equals to $\sum_{j=1}^{K} b_{i j} r_{j}=r_{i}+\sum_{j=1}^{K}\left(M_{i j}+L_{i j}\right) r_{j}$. A common factor $1 / \beta$ is introduced as the minimum fairness factor, so that for all nodes, $i$,

$$
\beta \sum_{j=1}^{K} a_{i j} r_{j} \geq \sum_{j=1}^{K} b_{i j} r_{j} .
$$

If equality holds for node $i$ in (4.16), then node $i$ can be viewed as making the maximum contribution to the network, normalized by the beneficial traffic it receives. If a strict inequality holds, it implies that node $i$ enjoys more benefit from the peering network than nodes with equality.

Consider now the pricing equations. For traffic originated from node $j$, represented by $r_{j}$, a total charge of $\sum_{i=1}^{K} b_{i j} p_{i}=p_{j}+\sum_{i=1}^{K}\left(M_{i j}+L_{i j}\right) p_{i}$ is incurred, and the economic benefit is represented by the amount $\sum_{i=1}^{K} a_{i j} p_{i}=p_{j}+\sum_{i=1}^{K} M_{i j} p_{i}$ Hence, one can define a maximum pricing efficiency ratio, $1 / \alpha$, so that for all nodes, $j$,

$$
\alpha \sum_{i=1}^{K} a_{i j} p_{i} \leq \sum_{i=1}^{K} b_{i j} p_{i}
$$

If equality holds for node $j$ in (4.17), the traffic it originates can be viewed as having the best pricing efficiency ratio in the network. If a strict inequality holds, it implies that the traffic originated from node $j$ does not enjoy the best pricing efficiency ratio. Following von Neumann, one adopts the following definition of an equilibrium solution.

Definition 6. An equilibrium solution is defined as a set of positive vectors, $\mathbf{r}$ and $\mathbf{p}$, satisfying equation (4.15) for some positive constants $\alpha$ and $\beta$, with the property that for any index $i, p_{i}=0$ if $\beta \sum_{j=1}^{K} a_{i j} r_{j}>\sum_{j=1}^{K} b_{i j} r_{j}$, and for all index $j, r_{j}=0$ if $\alpha \sum_{i=1}^{K} a_{i j} p_{i}<\sum_{i=1}^{K} b_{i j} p_{i}$.

To be more specific a set of positive vectors, $\mathbf{r}$ and $\mathbf{p}$, is an equilibrium solution if

1. Pricing efficiency indices at all nodes are bound above by $1 / \alpha$.

2. Fairness indices at all nodes are bounded below by $1 / \beta$.

3. Nodes with fairness indices strictly greater than $1 / \beta$, in a sense those nodes enjoying more routing service than then minimum level, are not allowed to collect fees; that is, prices at these nodes should be set to 0 . 
4. Data streams whose pricing efficiency are strictly less than $1 / \alpha$, in a sense data streams with economic efficiency less than $1 / \alpha$, are not allowed in the network; that is, rates of these streams should be set to 0 .

Therefore, these equilibrium solutions have physically meaning significance.

The following proposition follows from Neumann's equilibrium theorem (see [14]).

Proposition 7. If $\mathbf{A}$ and $\mathbf{B}$ are non-negative matrices and $\mathbf{A}+\mathbf{B} \gg 0$, then there exists a equilibrium solution to equation (4.15). Moreover, $1 / \alpha=1 / \beta$.

Note that the last equality in the proposition implies that $1 / \alpha$ and $1 / \beta$ are uniquely defined even though an equilibrium solution may not be uniquely defined. One can interpret $1 / \alpha$ as the maximum pricing efficiency that can be obtained an equilibrium solution, which is equal to $1 / \beta$ the minimum fairness index that can be guaranteed. For the source-destination model, refer to the equilibrium $1 / \alpha_{C}$ or $1 / \beta_{C}$ as the equilibrium source-destination-based index for the system. Similarly, one can define the equilibrium source-based index and the equilibrium destination-based index.

For illustration, one can revisit the previous example of a single routing node network. Numerical computations were carried out to track the equilibrium destinationbased index for this network as $x$ varies from 0 to 1 . As shown in Figure 4, the destination-fairness index exists up to the point 175/324. Beyond that point, only the equilibrium index exists, and is denoted by the lower dotted line. The upper dotted line shows the trajectory of the maximum fairness index value achieved by the equilibrium solutions. It is easy to check that before the bifurcation point, the equilibrium index and the fairness index are identical; both decrease monotonically as $x$ increases. This phenomenon is easy to explain. As $x$ increases, the amount of traffic routed through the single routing node increases, forcing its input traffic rate to decrease (so that the other two nodes would receive less useful traffic.) As a result, the fairness index also decreases. At the bifurcation point, traffic rate at the single routing node has already reached zero and cannot make any further compensation to counter the effect of increasing $x$. Beyond that point, the fairness indices cannot be balanced and the von Neumann index is a lower bound of all the indices, which is achieved by the single routing node.

5. Distributed Controller and Its Convergence. Given a set of target fairness indices, a natural question is how to ensure that the servers can achieve the targets by setting the input traffic rates correctly. A distributed control algorithm designed for this purpose is discussed here. The algorithm is based on the approach first proposed in [15] and further extended in [16]. It has some connection with the renowned "increase and decrease algorithms" for network control, [17]. (See [18] for 


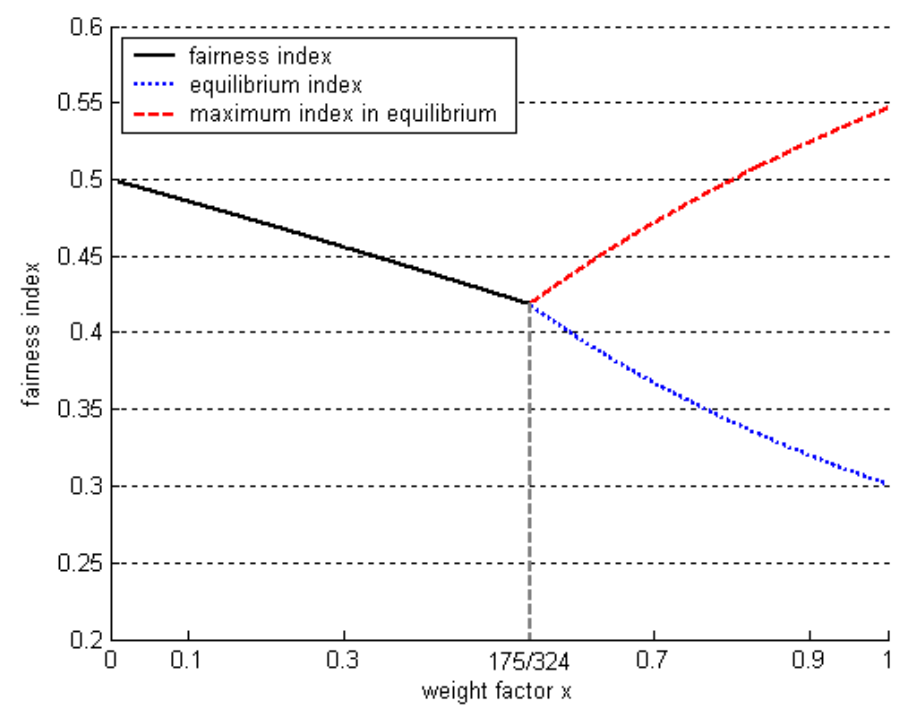

Figure 4

more references.)

For the algorithm to operate properly, network parameters, such as the traffic distribution matrix $\mathbf{M}$ and the transitory traffic matrix $\mathbf{L}$, need not be known to the servers. There is also no need for a central controller. Each node only has a local view of the network status: the incoming traffic rates consisting of the rate of traffic destined to it and the rate of transitory traffic, and its previous index value. Also, each node can only control its own traffic rate and has no direct control of other nodes.

In the following, a tracking algorithm for destination-fairness indices is presented, although the result can be extended to the other types of fairness indices. Let $T_{i j} r_{j}=$ $\left(M_{i j}+L_{i j}\right) r_{j}$ represent the total incoming traffic of node $i$ from node $j$, including both direct traffic destined to it and the transitory traffic passing by, then $\operatorname{Tr}$ is a column vector representing the total incoming data traffic rate of each node.

A set of positive performance targets, $\left\{\lambda_{1}, \ldots, \lambda_{K}\right\}$, is said to be feasible if there exists a positive traffic rate vector, $\left(\tilde{r}_{1}, \cdots, \tilde{r}_{K}\right)$, so that,

$$
\lambda_{i}=\frac{\sum_{j=1}^{K} M_{i j} \tilde{r}_{j}}{\tilde{r}_{i}+\sum_{j=1}^{K} T_{i j} \tilde{r}_{j}} .
$$

The proposed algorithm is based on a control law that adjusts the traffic rates by discrete levels, so that for server $i$, the levels are of the form $r_{i}(0) \delta_{i}^{k}$, for some step size $\delta_{i}>0$, an integer number $k$ (possibly negative,) and $r_{i}(0)>0$ represents the $i$-th component of the initial state. It follows that the achievable performance index levels 
are also discrete and the following result holds:

Lemma 2. Suppose that $1<\delta_{i} \leq \delta$ for $i=1, \ldots, K$ and $\delta^{2} \leq \varepsilon$. Let $\left(\tilde{r}_{1}, \cdots, \tilde{r}_{K}\right)$ be a state that achieves the performance targets, $\left\{\lambda_{1}, \ldots, \lambda_{K}\right\}$. Given any initial state, $\left(r_{1}(0), \ldots, r_{K}(0)\right)$, there exists a rate vector, $\mathbf{r}^{*}$, of the form, $\left(r_{1}(0) \delta_{1}^{i_{1}}, \ldots, r_{K}(0) \delta_{K}^{i_{K}}\right)$, such that the converged index for the $i$-th user, $\gamma_{i}^{*}$ satisfies

$$
\varepsilon^{-1} \lambda_{i} \leq \gamma_{i}^{*}=\frac{\sum_{j=1}^{K} M_{i j} r_{j}^{*}}{r_{i}^{*}+\sum_{j=1}^{K} T_{i j} r_{j}^{*}} \leq \varepsilon \lambda_{i} .
$$

Proof. It is clear that given any initial state, $\left(r_{1}(0), \ldots, r_{K}(0)\right)$, there exists a rate vector, $\mathbf{r}^{*}$, of the form, $\left(r_{1}(0) \delta_{1}^{i_{1}}, \ldots, r_{K}(0) \delta_{K}^{i_{K}}\right)$, such that $\delta^{-1} \tilde{r}_{i} \leq r_{i}^{*} \leq \delta \tilde{r}_{i}$. Then,

$$
\gamma_{i}^{*}=\frac{\sum_{j=1}^{K} M_{i j} r_{j}^{*}}{r_{i}^{*}+\sum_{j=1}^{K} T_{i j} r_{j}^{*}} \leq \frac{\sum_{j=1}^{K} M_{i j} \tilde{r}_{j} \delta}{\tilde{r}_{i} \delta^{-1}+\sum_{j=1}^{K} T_{i j} \tilde{r}_{j} \delta^{-1}} \leq \varepsilon \lambda_{i}
$$

The other inequality can be derived similarly.

Denote the destination-fairness index at iteration $n$ as

$$
\gamma_{i}(n)=\frac{\sum_{j=1}^{K} M_{i j} r_{j}(n)}{r_{i}(n)+\sum_{j=1}^{K} T_{i j} r_{j}(n)} .
$$

For positive targets, $\left\{\lambda_{1}, \ldots, \lambda_{K}\right\}$, the tri-state distributed control algorithm, is defined as

$$
r_{i}(n+1)=\left\{\begin{array}{lc}
r_{i}(n) \delta & \text { if } \gamma_{i}(n)>\varepsilon \lambda_{i} \\
r_{i}(n) \delta^{-1} & \text { if } \gamma_{i}(n)<\varepsilon^{-1} \lambda_{i} \\
r_{i}(n) & \text { else. }
\end{array}\right.
$$

If $\left\{\lambda_{1}, \ldots, \lambda_{K}\right\}$ is feasible, then under a suitable technical condition one can show that the tri-state algorithm converges in the sense that from any initial state, $\left(r_{1}(0)\right.$, $\left.\ldots, r_{K}(0)\right)$, in the positive orthant, the algorithm terminates at a state, $\left(r_{1}^{*}, \ldots, r_{K}^{*}\right)$, that satisfies equation (5.2). The proof of convergence is based on the approach first defined in [15]. However, due to the more complex nature of the model, the proof requires the following technical condition on the structure of the system parameters.

Technical Assumption: For $i=1, \ldots, K$, and all $j \neq i$ with $T_{i j} \neq 0$,

$$
\lambda_{i} \leq \frac{1}{\varepsilon} \cdot \frac{M_{i j}}{T_{i j}}
$$

Proposition 8. Assume technical condition (5.6) holds for a set of feasible targets. The trajectory of the distributed algorithm starting from an initial state in the positive orthant is bounded. 
To prove this, first define, as in [15], $a_{i}(n)$ at iteration $n$ by

$$
\mathbf{r}_{i}(n)=r_{i}^{*} \delta^{a_{i}(n)}
$$

Define $L(n)$ and $M(n)$ as $\max _{i}\left(a_{i}(n), 0\right)$ and $\max _{i}\left(-a_{i}(n), 0\right)$ respectively. The trajectory is bounded if $L(n)$ and $M(n)$ can be proven to be non-increasing. Details of this proof are described in the appendix.

Once the trajectory is shown to be bounded, the convergence result can be established if it can be shown that the trajectory does not exhibit any cycling behavior. To do this, the following definition from [15] is introduced.

Definition 7. A node, $i$, has a downward run with length $k, k>1$, from time $m$ to $n(m<n)$, if the following relations hold:

1. $r_{i}(m-1)=\delta^{-1} r_{i}(m)$,

2. $r_{i}(m)=\delta^{k} r_{i}(n)$.

Proposition 9. Assume technical condition (5.6) holds for a set of feasible targets, $\left\{\lambda_{1}, \ldots, \lambda_{K}\right\}$, and that $\varepsilon$ is large enough so that

$$
\varepsilon^{2} \min _{j, M_{i j} \neq 0}\left(M_{i j} / T_{i j}\right) \geq \max _{j, M_{i j} \neq 0}\left(M_{i j} / T_{i j}\right)
$$

for all $i$. Then, the trajectory of the tri-state algorithm, starting from an initial state in the positive orthant, cannot be asymptotically periodic with a minimum period larger than 1.

Details of the proof can be found in the appendix.

Theorem 1. Assume technical assumption (5.6) holds for a set of feasible targets, $\left\{\lambda_{1}, \ldots, \lambda_{K}\right\}$. Assume that $\delta^{2} \leq \varepsilon$ and for all $i, \varepsilon^{2} \min _{j, M_{i j} \neq 0}\left(M_{i j} / T_{i j}\right) \geq$ $\max _{j, M_{i j} \neq 0}\left(M_{i j} / T_{i j}\right)$. Under the tri-state distributed control algorithm (5.5), from any initial state in the positive orthant, the data traffic rate at each node converges to a state, whose performance index satisfies equation (5.2). That is

$$
\lim _{n \rightarrow \infty} r_{i}(n)=r_{i}^{*}, \text { and } \varepsilon^{-1} \lambda_{i} \leq \gamma_{i}^{*}=\frac{\sum_{j=1}^{K} M_{i j} r_{j}^{*}}{r_{i}^{*}+\sum_{j=1}^{K} T_{i j} r_{j}^{*}} \leq \varepsilon \lambda_{i} .
$$

Proof. Proposition 8 implies that for any initial state in the positive orthant the trajectory under the distributed algorithm is bounded. Since the feasible states are discrete, the number of reachable state is finite. Hence, the algorithm will either converge to a fixed point or to a cycle. From Proposition 9, the latter case is ruled out. Since the system converges to a fix point, equation (5.5) must hold.

6. Numerical Results. For a demonstration of the convergence properties of the distributed control algorithm, consider a five-node network with 


$$
\begin{aligned}
\mathbf{M} & =\left[\begin{array}{ccccc}
0 & 0.2418 & 0.2304 & 0.2341 & 0.2549 \\
0.2638 & 0 & 0.2577 & 0.2595 & 0.2520 \\
0.2418 & 0.2658 & 0 & 0.2532 & 0.2329 \\
0.2377 & 0.2534 & 0.2583 & 0 & 0.2602 \\
0.2566 & 0.2390 & 0.2536 & 0.2531 & 0
\end{array}\right] \\
\mathbf{L} & =\left[\begin{array}{ccccc}
0 & 0.5212 & 0.5291 & 0.5266 & 0.5123 \\
0.5061 & 0 & 0.5103 & 0.5091 & 0.5142 \\
0.5213 & 0.5048 & 0 & 0.5134 & 0.5274 \\
0.5240 & 0.5133 & 0.5099 & 0 & 0.5086 \\
0.5111 & 0.5232 & 0.5131 & 0.5135 & 0
\end{array}\right] .
\end{aligned}
$$

The feasible performance targets are $\left\{\lambda_{1}=\lambda_{2}=\ldots=\lambda_{K}=0.2462\right\}, \delta=1.01$ and $\varepsilon=1.021$. One can check that condition (5.6) is satisfied. Although condition (5.8) is not satisfied, simulation results in Figure 5 show that starting from a randomly selected initial state in the positive orthant, the trajectory under the tri-state control algorithm converges after 82 iterations to $\gamma^{*}=\left\{\begin{array}{lllll}0.2414 & 0.2501 & 0.2447 & 0.2483 & 0.2462\end{array}\right\}$, which satisfies the condition $\varepsilon^{-1} \lambda_{i} \leq \gamma_{i}^{*} \leq \varepsilon \lambda_{i}$.

More simulations were taken for a variety of network topologies as summarized in Table 1 , (with $\delta=1.01$ and $\varepsilon=1.03$ ).

\begin{tabular}{|c|c|c|c|c|c|c|c|}
\hline Simulation No. & 1 & 2 & 3 & 4 & 5 & 6 & 7 \\
\hline No. of Nodes & 5 & 6 & 6 & 7 & 7 & 8 & 8 \\
\hline Fairness Index & 0.2462 & 0.2337 & 0.2344 & 0.1940 & 0.1892 & 0.1592 & 0.1583 \\
\hline Converged Index & 0.2448 & 0.2326 & 0.2366 & 0.1944 & 0.1911 & 0.1577 & 0.1578 \\
& 0.2446 & 0.2358 & 0.2357 & 0.1930 & 0.1908 & 0.1579 & 0.1578 \\
& 0.2485 & 0.2314 & 0.2351 & 0.1958 & 0.1875 & 0.1607 & 0.1599 \\
& 0.2437 & 0.2326 & 0.2323 & 0.1954 & 0.1873 & 0.1606 & 0.1568 \\
& 0.2485 & 0.2358 & 0.2329 & 0.1922 & 0.1892 & 0.1585 & 0.1567 \\
& & 0.2360 & 0.2328 & 0.1959 & 0.1894 & 0.1585 & 0.1598 \\
& & & & 0.1925 & 0.1878 & 0.1600 & 0.1592 \\
& & & & & & 0.1606 & 0.1599 \\
\hline No. of Iterations & 266 & 122 & 324 & 207 & 219 & 243 & 165 \\
\hline
\end{tabular}

Table 1

These results show the tri-state control algorithm has good convergence properties. However, it assumes the feasible performance targets are known a priori. An invalid target value will result in a process that does not converge as shown in Figure 
6 , which is based on the previously introduced 5 -node network but with target value set to 0.22 .

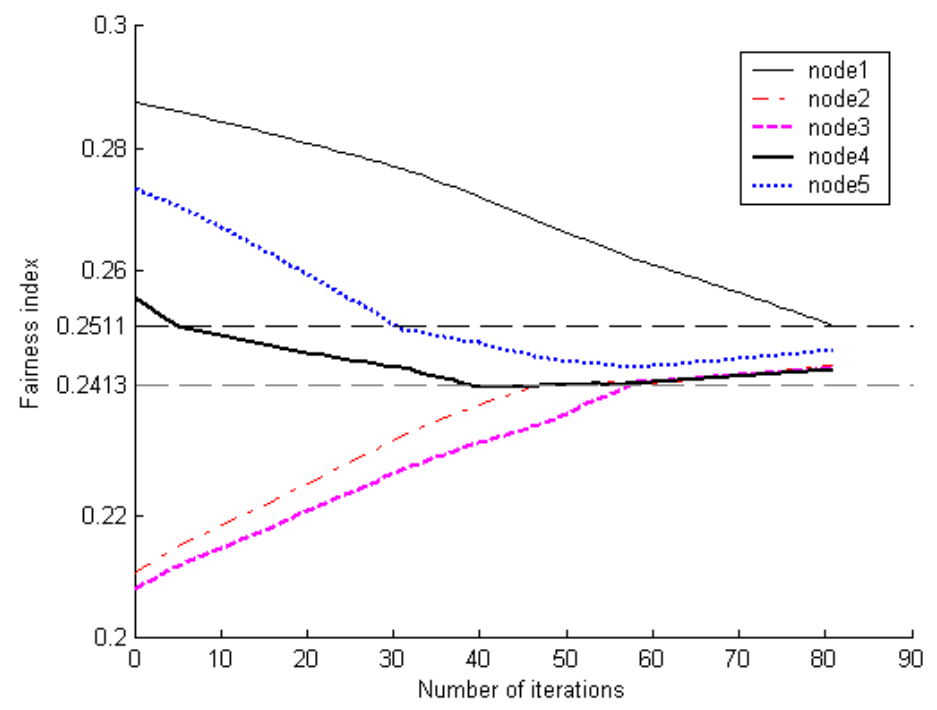

Figure 5

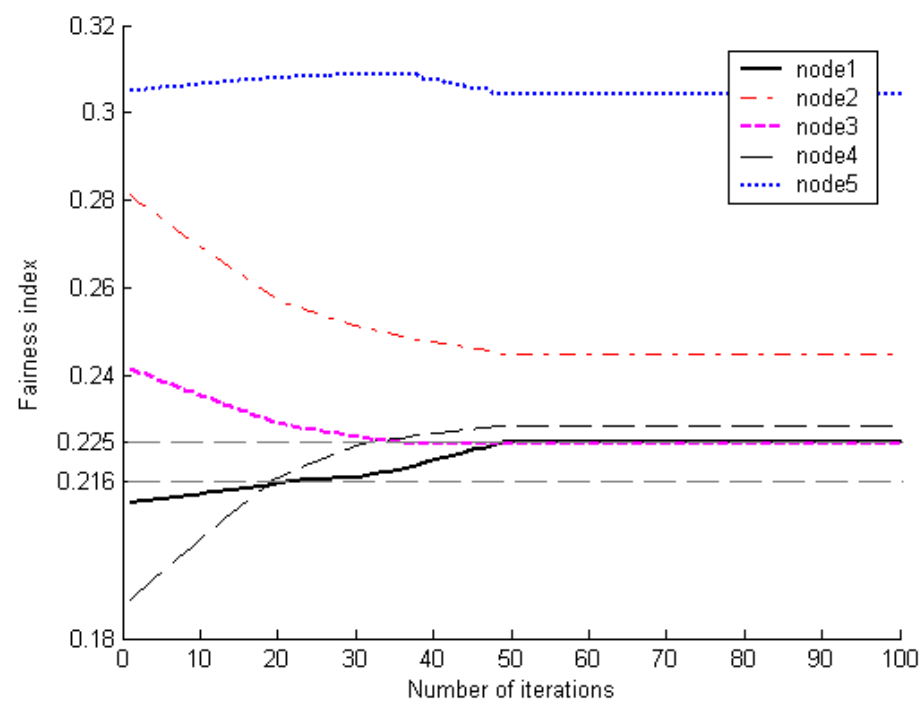

Figure 6

In practice it is difficult to determine whether a set of performance targets is 
feasible or not, especially for large networks. Moreover, for the case where one is interested in achieving absolutely fair solutions, the value of the absolutely fair index may not be known. However, it is possible to design a heuristic algorithm for convergence to absolutely fair solutions. The basic idea is to start the tri-state algorithm based on an initial estimate of the index and adjust the target value if convergence is not achieved within a pre-set duration. This heuristic algorithm is only partially distributed in the sense that it needs a central controller to update the target values. By periodically updating information for all the nodes, the algorithm can converge to an absolutely fair solution if such a solution exists for the network. The following is a description of the heuristic algorithm.

Heuristic Iterative Algorithm:

Set initial values: initial values for $\delta$ and $\varepsilon$ are set to relatively large values, $\left(\delta^{2} \leq \varepsilon\right)$, initial target value $\gamma_{0}$ and traffic rates $\mathbf{r}_{0}$ are also set.

Start the tri-state algorithm until the trajectory converges to a band defined by the currently set target value or remains unchanged outside the target band after a maximal iteration number $\kappa$.

If the tri-state algorithm stops for reaching the maximum iteration limit, the nodes inform their current target value to the central controller. The mean of the target values is set as the target value for the next cycle. Values for $\delta$ and $\varepsilon$ are unchanged.

If the tri-state algorithm converges to within the band defined by the currently set target value, $\delta$ and $\varepsilon$ are decreased for the next cycle. (One approach is to set the value to the square root of the current value.) Target value is unchanged.

The cycles are repeated until $\delta$ and $\varepsilon$ are decreased to predefined acceptable value $\delta_{0}$ and $\varepsilon_{0}$ and convergence to the currently set target value band is achieved.

To illustrate, consider the previous example with an initial target value as $\gamma_{0}=0.4$. Obviously this performance target is not a feasible value, so convergence cannot be obtained by simply applying the tri-state algorithm. A simulation study was carried out using the heuristic algorithm by setting initially $\delta=1.19, \varepsilon=1.41$ and $\kappa=8$.

For target value set as $\gamma_{0}=0.4$, the tri-state algorithm did not converge. However, the fairness value trajectories remained constant after a few iterations; some of index values are outside of the target band. The maximum iteration limit was reached as shown in Figure 7. The target was reset to the mean value of current indices for the next cycle and the whole process was repeated. When convergence was observed, $\delta$ and $\varepsilon$ were respectively decreased by setting them to the square root of their current value. After two cycles, the target value reached 0.2453 with $\delta=1.0043$ and $\varepsilon=1.0905$. 


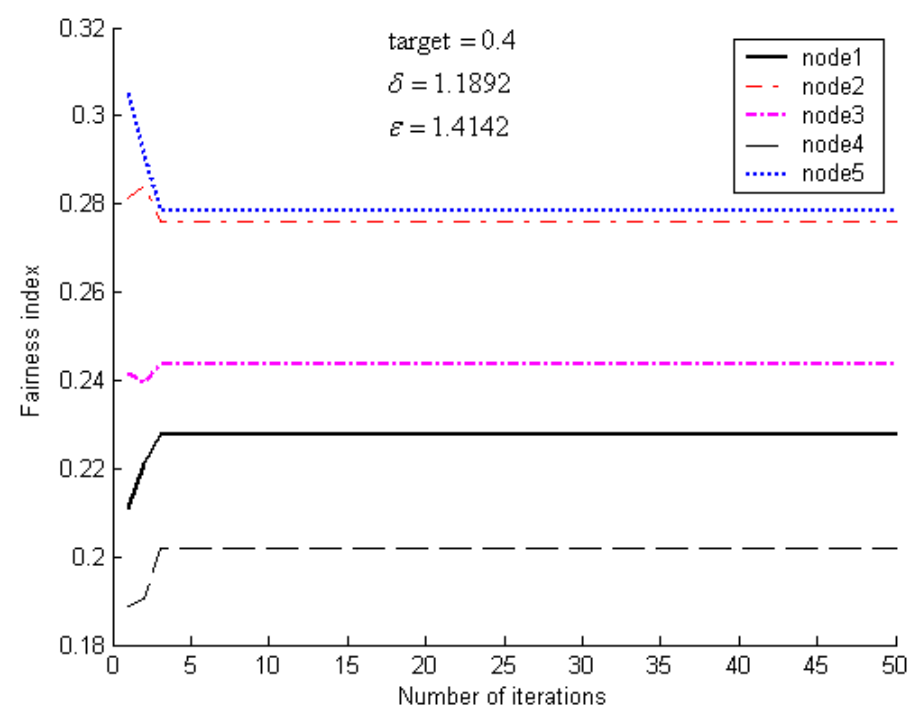

Figure 7

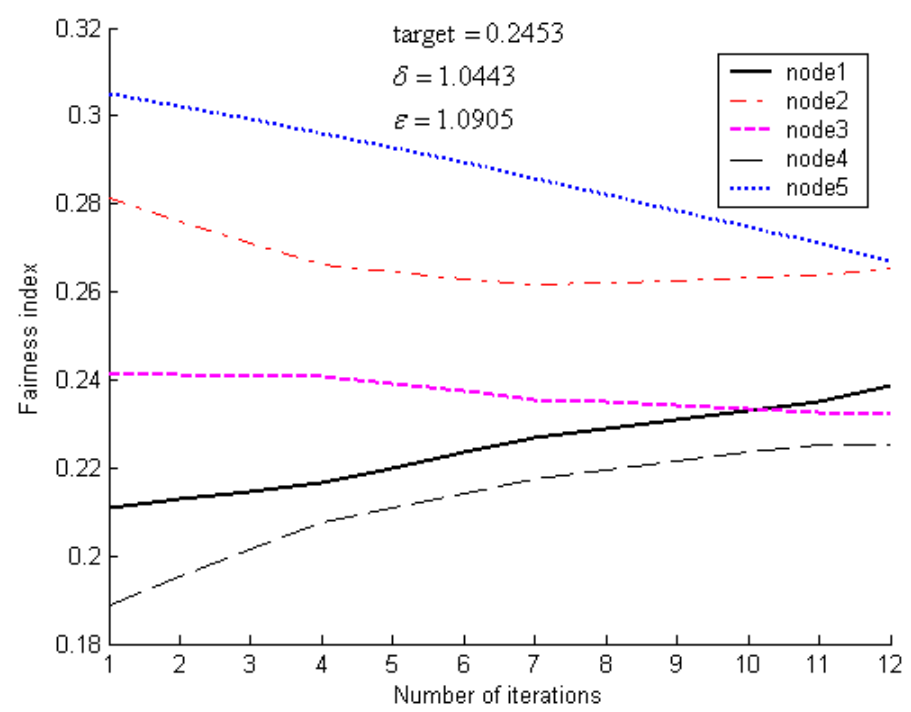

Figure 8

The tri-state algorithm converged to the target value this time as showed in Figure 8. By the 8 th cycles, $\delta$ and $\varepsilon$ dropped to the pre-defined value $\delta_{0}$ and $\varepsilon_{0}$, and the achieved target value was in the corresponding band defined by the actual system fairness index $\gamma^{*}$ as showed in Figure 9. 


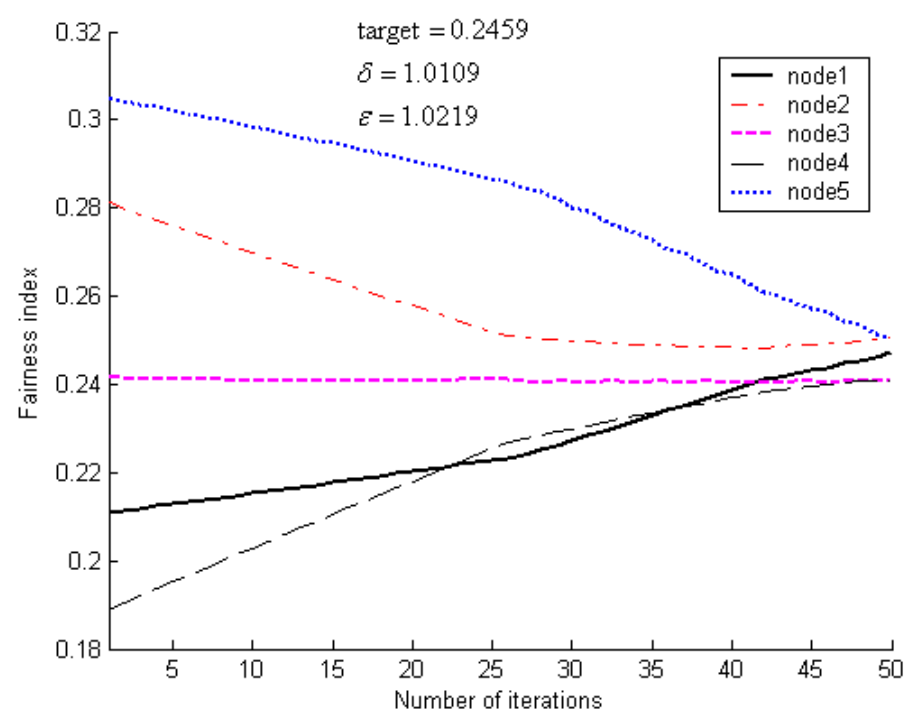

Figure 9

7. Conclusion . In this paper a new fairness concept for a network is discussed. Depending on the objective, one can formulate various network control models based on the class of fairness indices introduced. One particular issue examined here is the concept of absolute fairness, which can be related to the von Neumann equilibrium by introducing a pricing duality. The issue of using a distributed control algorithm to track fairness performance indices is also examined. A low complexity, low data rate algorithm is presented and its convergence properties is studied. Heuristic extension of the algorithm to practical situations is also explained.

Acknowledgment: The authors would like to thank Professor Dah Ming Chiu for helpful discussions on the paper, which helped us reformulate the definition of fairness index from the form stated in [2] to the form stated here.

\section{REFERENCES}

[1] A. Oram, Peer-to-Peer Harnessing the Power of Disruptive Technologies, O'Reilly \& Associates, March 2001.

[2] W. S. Wong, Fairness issues in peer-to-peer networks, Proceedings of the IEEE 2002 Conference on Decision and Control, Las Vegas, Nevada, December 2002.

[3] W. S. Wong and Fengunn Li, A fairness index for communication networks, in: New Directions in Control and Applications, Lecture Notes in Control and Information Sciences, Vol 321, Anders Lindquist et al, ed., Springer-Verlag, 2005.

[4] Jean-Yves Le Boudec, Rate adaptation, congestion control and fairness: a tutorial, http://ica1www.epfl.ch/PS_files/LEB3132.pdf.

[5] F. Kelly, Charging and Rate Control for Elastic Traffic, European Transactions on Telecom- 
munications, 8(1997), pp. 33-37.

[6] R. J. Gibbens and F. P. Kelly, Resource Pricing and the Evolution of Congestion Control, Automatica, 35(1999), pp. 1969-1985.

[7] F. Kelly, A, Maulloo, and D. Tan, Rate Control for Communication Networks: Shadow Price Proportional Fairness and Stability, J. Oper. Res. Soc., 49(1998), pp. 237-252.

[8] R. Mazumdar, L. G. Mason, and C. Douligeris, Fairness in Network Optimal Flow Control: Optimality of Product Forms, IEEE Transactions of Communications, 39(1991), pp. 775782.

[9] J. Mo and J. Walrand, Fair End-to-End Window-Based Congestion Control, IEEE/ACM Transactions on Networking, 8(2000), pp. 556-567.

[10] A. Berman and R. J. Plemmons, Non-negative Matrices in the Mathematical Sciences, SIAM Edition, Society for Industrial and Applied Mathematics, Philadelphia, 1994.

[11] A. TAng, J. Wang, And S. H. Low, Is Fair Allocation Always Inefficient, Proceedings IEEE INFOCOM, Hong Kong, March 2004.

[12] O. L. Mangasarian, Perron-Frobenius Properties of $A x-\lambda B x$, Journal of Mathematical Analysis and Applications, 36(1971), pp. 86-102.

[13] E. Seneta, Non-negative Matrices and Markov Chains, Second Edition, Springer-Verlag, New York, 1981.

[14] J. V. Neumann, A Model of General Economic Equilibrium, The Review of Economic Studies, 13:1(1945-1946), pp. 1-9.

[15] C. W. Sung And W. S. Wong, A Distributed Fixed-Step Power Control Algorithm with Quantization and Active Link Quality Protection, IEEE Transactions on Vehicular Technology, 48(1999), pp. 553-562.

[16] W. S. Wong and C. W. Sung, Robust Convergence of Low Data Rate Distributed Controllers, IEEE Transactions on Automatic Control, 49:1(2004), pp. 82-87.

[17] D. Chiu And R. Jain, Analysis of the Increase and Decrease Algorithms for Congestion Avoidance in Computer Networks, Comput. Networks ISDN Syst., 17(1989), pp. 1-14.

[18] S. LOW AND D. LAPSLEY, Optimization flow control-I: basic algorithm and convergence, IEEE/ACM Transactions on Networking, 7:6(1999), pp. 861-874.

[19] D. Serre, Matrices: Theory and Applications, New York: Springer-Verlag, 2002. 
Appendix. Proof of Proposition 4:

Assume that $\mathbf{r}$ is a positive solution to (3.7). From (3.7) it follows that

$$
\left(1 / \gamma_{C}-1+c\right) \mathbf{e}^{T}(\mathbf{I}+\mathbf{M}) \mathbf{r}=2\left(1 / \gamma_{C}-1+c\right) \mathbf{e}^{T} \mathbf{r}=c K \cdot \mathbf{e}^{T} \mathbf{r} .
$$

Statement 1 then follows since $\mathbf{e}^{T} \mathbf{r} \neq 0$. To find a strictly positive solution to equation (3.7) rewrite it as

$$
K / 2(\mathbf{I}+\mathbf{M}) \mathbf{r}=\mathbf{K r} .
$$

Note that any solution to (2) leads to a solution to

$$
\left(\mathbf{I}+\mathbf{N}_{s}\right) \mathbf{r}=(2 / K-s) \mathbf{K r},
$$

and vice versa. We claim that $\rho\left(N_{s}\right)<1$. To prove this, note that

$$
\begin{aligned}
\operatorname{det}[t \mathbf{I}-(\mathbf{M}-s \mathbf{K})] & =\operatorname{det}[t \mathbf{I}-\mathbf{M}] \operatorname{det}\left[\mathbf{I}+s(t \mathbf{I}-\mathbf{M})^{-1} \mathbf{K}\right] \\
& =\operatorname{det}[t \mathbf{I}-\mathbf{M}]\left[1+s \mathbf{e}^{T}(t \mathbf{I}-\mathbf{M})^{-1} \mathbf{e}\right] .
\end{aligned}
$$

The second equality follows from a well-known formula of the characteristic function of a matrix, $\operatorname{det}(t \mathbf{I}-\mathbf{M})$ (see for example [19].). For large enough $t$,

$$
\begin{aligned}
\operatorname{det}[t \mathbf{I}-(\mathbf{M}-s \mathbf{K})] & =\operatorname{det}[t \mathbf{I}-\mathbf{M}]\left[1+s \mathbf{e}^{T}(t \mathbf{I}-\mathbf{M})^{-1} \mathbf{e}\right] \\
& =\operatorname{det}[t \mathbf{I}-\mathbf{M}]\left[1+\frac{s}{t} \mathbf{e}^{T}\left(\mathbf{I}-\frac{\mathbf{M}}{t}\right)^{-1} \mathbf{e}\right] \\
& =\operatorname{det}[t \mathbf{I}-\mathbf{M}]\left[1+\frac{s}{t} \mathbf{e}^{T}\left(1+\frac{\mathbf{M}}{t}+\frac{\mathbf{M}^{2}}{t^{2}}+\cdots\right) \mathbf{e}\right] \\
& =\operatorname{det}[t \mathbf{I}-\mathbf{M}] \frac{t-1+s K}{t-1} .
\end{aligned}
$$

Since $\operatorname{det}[t \mathbf{I}-(\mathbf{M}-s \mathbf{K})]$ is a polynomial in $t$, and since $\operatorname{det}[t \mathbf{I}-\mathbf{M}]$ has a root at $t=1$, it follow that equation (6) holds for all $t$. Hence, the eigenvalues of $\mathbf{M}-s \mathbf{K}$ is same as those of $\mathbf{M}$, except that the eigenvalue 1 is replaced by $1-s K$. Note that $|1-s K|<1$. Since $\mathbf{M}$ is assumed to be a primitive matrix with $\rho(\mathbf{M})=1$, it follows that all eigenvalues of $\mathbf{M}-s \mathbf{K}$ (that is all eigenvalues of $\mathbf{M}$ other than 1,) are less than 1 (see for example [10]). Hence $\rho\left(N_{s}\right)<1$, and the series $\mathbf{I}-\mathbf{N}_{s}+\mathbf{N}_{s}^{2}-\cdots$ is convergent. Moreover, by definition of $s,-\mathbf{N}_{s}$ is a non-negative matrix. Hence, it is easy to check that equation (3) defines a strictly positive vector. By substituting the solution (3.9), the right hand side of (3) evaluates to e. On the other hand,

$$
\begin{aligned}
(2 / K-s) \mathbf{K}\left(\mathbf{I}+\mathbf{N}_{s}\right)^{-1} \mathbf{e} & =(2 / K-s) \mathbf{K}\left(\mathbf{I}-\mathbf{N}_{s}+\mathbf{N}_{s}^{2}-\cdots\right) \mathbf{e} \\
& =(2 / K-s)\left(\mathbf{K}-(1-s K) \mathbf{K}+(1-s K)^{2} \mathbf{K}-\cdots\right) \mathbf{e} \\
& =(2-s K)\left(1-(1-s K)+(1-s K)^{2}-\cdots\right) \mathbf{e}=\mathbf{e} .
\end{aligned}
$$


Hence, (3.9) is a strictly positive solution (3.7) that is unique up to multiplication by a positive scalar.

\section{Proof of Proposition 5:}

Assume that $\mathbf{r}$ is a positive solution to (3.8). From (3.8) it follows that

$$
1 / \gamma_{D} e^{T} \mathbf{M r}+(c-1) e^{T}(\mathbf{I}+\mathbf{M}) \mathbf{r}=\left(1 / \gamma_{D}+2(c-1)\right) e^{T} \mathbf{r}=c K \cdot e^{T} \mathbf{r} .
$$

Since $\mathbf{e}^{T} \mathbf{r} \neq 0$, it is obvious that

$$
1 / \gamma_{D}+2(c-1)=c K
$$

and statement 1 follows. To establish statement 2, note that (3.8) can be re-written as

$$
\left(\frac{1-c+c K}{1-c} \mathbf{M}-\frac{c}{1-c} K\right) \mathbf{r}=I \mathbf{r}
$$

By the assumption on $\mathbf{M}, \frac{1-c+c K}{1-c} \mathbf{M}-\frac{c}{1-c} K$ is a strictly positive matrix, so that it has a strictly positive eigenvector, $\mathbf{r}$, which is unique up to multiplication by a positive scalar.

\section{Proof of Proposition 6:}

Considering $L^{2}=0$, and

$$
I=I^{2}-L^{2}=(I+L)(I-L)
$$

for this particular network architecture, equation (2.4) can be re-written in the following way with $\beta=1 / \gamma_{O}-1>0$.

$$
\left(\frac{1}{\gamma_{O}}-1\right)(I-L) M r=\beta\left[\begin{array}{ll}
-\mathbf{L}_{12}^{T} \mathbf{M}_{21} & \mathbf{M}_{12}^{T}-L_{12}^{T} M_{22} \\
M_{21} & M_{22}
\end{array}\right]\left[\begin{array}{l}
r_{1} \\
r_{2}
\end{array}\right]=\left[\begin{array}{l}
r_{1} \\
r_{2}
\end{array}\right] .
$$

Note that for any $\beta$, satisfying $0<1 / \beta<\rho\left(\mathbf{M}_{22}\right)$ and any positiver $r_{1}$, the $K$ 1 dimensional vector $r_{2}=\left(I / \beta-M_{22}\right)^{-1} M_{21} r_{1}$ is positive. Moreover, the vector $\left[\begin{array}{l}r_{1} \\ r_{2}\end{array}\right]$ is a positive solution to equation (2.4) if the following equality holds for $0<1 / \beta<\rho\left(\mathbf{M}_{22}\right)$ :

$$
\begin{aligned}
\frac{1}{\beta} & =\left(M_{12}^{T}-L_{12}^{T} M_{22}\right)\left(\frac{I}{\beta}-M_{22}\right)^{-1} M_{21}-L_{12}^{T} M_{21} \\
& =\left(M_{12}^{T}-L_{12}^{T} / \beta\right)\left(\frac{I}{\beta}-M_{22}\right)^{-1} M_{21} .
\end{aligned}
$$

Consider the last expression of equation (14) as a function of $\beta$ as denote it as $f(\beta)$. Since $f(\beta)$ has a pole at $\beta=\rho\left(M_{22}\right)^{-1}$, equation (14) has a positive solution if $f(\beta)$ tends to infinity when $\beta$ approaching $1 / \rho\left(M_{22}\right)^{-1}$. 
To prove this, first define $y(\beta)=\left(1 / \beta-\rho\left(M_{22}\right)\right)\left(I / \beta-M_{22}\right)^{-1}$. Since $\mathbf{M}$ is irreducible so is $M_{22}$. It is well known that $y\left(1 / \rho\left(M_{22}\right)\right)$ is a matrix whose columns are positive eigenvectors of $M_{22}$ (see for example [13]). Hence $\mathbf{z}=y\left(1 / \rho\left(M_{22}\right)\right) M_{21}$ is a positive eigenvector of $M_{22}$. By assumption (3.13), $\left(1 / \beta-\rho\left(M_{22}\right)\right) f(\beta)$ is positive as $\beta$ approaching $1 / \rho\left(M_{22}\right)^{-1}$.

\section{Proof of Proposition 8:}

At iteration $n$, define $a_{i}(n)$ by

$$
\mathbf{r}_{i}(n)=r_{i}^{*} \delta^{a_{i}(n)} .
$$

Let $L(n)=\max _{i}\left(a_{i}(n), 0\right)$. Assume without lost of generality, the maximum is achieved by node 1 at time $n$. For $k=1, \ldots, K$, define

$$
z_{k}^{L}(n)=\frac{\sum_{j=2}^{k} M_{1 j} r_{j}^{*} \delta^{L(n)}+\sum_{j=k+1}^{K} M_{1 j} r_{j}(n)}{r_{1}^{*} \delta^{L(n)}+\sum_{j=2}^{k} T_{1 j} r_{j}^{*} \delta^{L(n)}+\sum_{j=k+1}^{K} T_{1 j} r_{j}(n)} .
$$

Notice that $z_{K}^{L}(n)=\gamma_{1}^{*} \leq M_{1 j} / T_{1 j}$, for all $j \neq 1$ with $T_{1 j} \neq 0$. We claim that for $k=2, \ldots, K$,

$$
z_{k-1}^{L}(n) \leq z_{k}^{L}(n)
$$

To show this, assume the inequality holds from $K$ to $k$. If $r_{k}(n)=r_{k}^{*} \delta^{L(n)}$, then the inequality in (15) is an equality. If not, by the definition of $L(n)$, this implies $r_{k}(n)<r_{k}^{*} \delta^{L(n)}$. Let

$$
\begin{gathered}
a=r_{1}^{*} \delta^{L(n)}+\sum_{j=2}^{k-1} T_{1 j} r_{j}^{*} \delta^{L(n)}+\sum_{j=k+1}^{K} T_{1 j} r_{j}(n), \\
b=\sum_{j=2}^{k-1} M_{1 j} r_{j}^{*} \delta^{L(n)}+\sum_{j=k+1}^{K} M_{1 j} r_{j}(n) .
\end{gathered}
$$

Then

$$
z_{k-1}^{L}(n)=\frac{b+M_{1 k} r_{k}(n)}{a+T_{1 k} r_{k}(n)}, \quad z_{k}^{L}(n)=\frac{b+M_{1 k} r_{k}^{*} \delta^{L(n)}}{a+T_{1 k} r_{k}^{*} \delta^{L(n)}} .
$$

Consider the difference $z_{k}^{L}(n)-z_{k-1}^{L}(n)$. This term is non-negative if and only if

$$
\begin{aligned}
& {\left[b+M_{1 k} r_{k}^{*} \delta^{L(n)}\right]\left[a+T_{1 k} r_{k}(n)\right]-\left[b+M_{1 k} r_{k}(n)\right]\left[a+T_{1 k} r_{k}^{*} \delta^{L(n)}\right] } \\
= & {\left[a M_{1 k}-b T_{1 k}\right]\left[r_{k}^{*} \delta^{L(n)}-r_{k}(n)\right] \geq 0 . }
\end{aligned}
$$

Since $z_{k}^{L}(n)=\frac{b+M_{1 k} r_{k}^{*} \delta^{L(n)}}{a+T_{1 k} r_{k}^{*} \delta^{L(n)}} \leq z_{K}^{L}(n) \leq \frac{M_{1 k}}{T_{1 k}}$, it follows that $\frac{b}{a} \leq \frac{M_{1 k}}{T_{1 k}}$, and then $z_{k}^{L}(n) \geq z_{k-1}^{L}(n)$. So by induction, equation (15) holds. It follows that $\gamma_{1}(n)=$ 
$z_{1}^{L}(n) \leq z_{K}^{L}(n)=\gamma_{1}^{*} \leq \lambda_{1} \varepsilon$, therefore $a_{1}(n+1) \leq a_{1}(n)$. For the case $a_{i}(n)<L(n)$, it is clear that $a_{i}(n+1) \leq L(n)$. Hence, $L(n)$ is non-increasing.

Consider now $M(n)=\max _{i}\left(-a_{i}(n), 0\right)$. Again assume without lost of generality, the maximum is achieved by node 1 at time $n$. Now for $k=1, \ldots, K$, define

$$
z_{k}^{M}(n)=\frac{\sum_{j=2}^{k} M_{1 j} r_{j}^{*} \delta^{-M(n)}+\sum_{j=k+1}^{K} M_{1 j} r_{j}(n)}{r_{1}^{*} \delta^{-M(n)}+\sum_{j=2}^{k} T_{1 j} r_{j}^{*} \delta^{-M(n)}+\sum_{j=k+1}^{K} T_{1 j} r_{j}(n)} .
$$

As before, assume that without lost of generality the maximum is achieved by $i=$ 1 , one can show that $M(n)$ is also non-increasing. To establish this, first re-label the indices so that

$$
\frac{M_{1 K}}{T_{1 K}} \leq \cdots \leq \frac{M_{13}}{T_{13}} \leq \frac{M_{12}}{T_{12}}
$$

Notice that $z_{K}^{M}(n)=\gamma_{1}^{*} \leq M_{1 j} / T_{1 j}$, for all $j \neq 1$ with $T_{1 j} \neq 0$. One can show that for $k=2, \ldots, K$, the following inequalities (22) and (23) hold.

$$
\begin{aligned}
& z_{k-1}^{M}(n) \geq z_{k}^{M}(n), \\
& z_{k}^{M}(n) \leq M_{1 k} / T_{1 k} .
\end{aligned}
$$

To prove the claim, assume that inequalities (22) and (23) hold all for values from $K$ to $k$. If $r_{k}(n)=r_{k}^{*} \delta^{-M(n)}$, then inequality (22) follows. If not, by the definition of $M(n)$, this implies $r_{k}(n)>r_{k}^{*} \delta^{-M(n)}$, Let

$$
\begin{aligned}
c & =r_{1}^{*} \delta^{-M(n)}+\sum_{j=2}^{k-1} T_{1 j} r_{j}^{*} \delta^{-M(n)}+\sum_{j=k+1}^{K} T_{1 j} r_{j}(n), \\
d & =\sum_{j=2}^{k-1} M_{1 j} r_{j}^{*} \delta^{-M(n)}+\sum_{j=k+1}^{K} M_{1 j} r_{j}(n) .
\end{aligned}
$$

Then

$$
\begin{aligned}
z_{k-1}^{M}(n) & =\frac{d+M_{1 k} r_{k}(n)}{c+T_{1 k} r_{k}(n)}, \\
z_{k}^{M}(n) & =\frac{d+M_{1 k} r_{k}^{*} \delta^{-M(n)}}{c+T_{1 k} r_{k}^{*} \delta^{-M(n)}} .
\end{aligned}
$$

Consider the difference $z_{k}^{M}(n)-z_{k-1}^{M}(n)$. This term is non-positive if and only if

$$
\begin{aligned}
& {\left[d+M_{1 k} r_{k}^{*} \delta^{-M(n)}\right]\left[c+T_{1 k} r_{k}(n)\right]-\left[d+M_{1 k} r_{k}(n)\right]\left[c+T_{1 k} r_{k}^{*} \delta^{-M(n)}\right] } \\
= & {\left[c M_{1 k}-d T_{1 k}\right]\left[r_{k}^{*} \delta^{-M(n)}-r_{k}(n)\right] \leq 0 . }
\end{aligned}
$$

Since $z_{k}^{M}(n)=\frac{d+M_{1 k} r_{k}^{*} \delta^{-M(n)}}{c+T_{1 k} r_{k}^{*} \delta^{-M(n)}} \leq \frac{M_{1 k}}{T_{1 k}}$, it follows that $\frac{d}{c} \leq \frac{M_{1 k}}{T_{1 k}}$. Hence $z_{k}^{M}(n) \leq$ $z_{k-1}^{M}(n)$ and 


$$
z_{k-1}^{M}(n)=\frac{d+M_{1 k} r_{k}(n)}{c+T_{1 k} r_{k}(n)} \leq \frac{M_{1 k}}{T_{1 k}} \leq \frac{M_{1, k-1}}{T_{1, k-1}}
$$

So, by induction inequality (22) holds. It follows that $\gamma_{1}(n)=z_{1}^{M}(n) \geq z_{K}^{M}(n)=$ $\gamma_{1}^{*} \geq \varepsilon^{-1} \lambda_{1}$. Therefore $a_{1}(n+1) \geq a_{1}(n)$. For the case $a_{i}(n)>-M(n)$, it is clear that $a_{i}(n+1) \geq-M(n)$. Hence, $M(n)$ is also non-increasing. This concludes the proof that the trajectory is bounded.

\section{Proof of Proposition 9:}

To prove proposition 9, one first establish the claim that if node $i$ has a run with length $k$ from time $m$ to $n$, then there is a node $j$ and a time instance, $l, m<l<n$, such that

$$
r_{j}(m-1) \geq \delta^{k+1} r_{j}(l)
$$

Assume without lost of the generality, this maximum run is achieved by node $i=1$, and $M_{1 j}$ for $2 \leq j \leq K$ are all non-zero. By the definition of a run, there is a time instance, $l, m<l<n$, such that

$\gamma_{1}^{*} \varepsilon^{-1}>\gamma_{1}^{(l)}$ and $r_{1}(l)=\delta r_{1}(n) .(29)$

Since the target is feasible, $\gamma_{1}^{(i)}>0$ for any $i$ and

$$
\gamma_{1}^{*} \varepsilon^{-1}>\gamma_{1}^{(l)}=\gamma_{1}^{(m-1)} \frac{\gamma_{1}^{(l)}}{\gamma_{1}^{(m-1)}}>\gamma_{1}^{*} \varepsilon \frac{\gamma_{1}^{(l)}}{\gamma_{1}^{(m-1)}} .
$$

It follows that

$$
\varepsilon^{2}<\frac{\gamma_{1}^{m-1}}{\gamma_{1}^{l}}=\frac{r_{1}(l)+\sum_{j=2}^{K} T_{1 j} r_{j}(l)}{r_{1}(m-1)+\sum_{j=2}^{K} T_{1 j} r_{j}(m-1)} \cdot \frac{\sum_{j=2}^{K} M_{1 j} r_{j}(m-1)}{\sum_{j=2}^{K} M_{1 j} r_{j}(l)} .
$$

This can be rewritten as

$$
\frac{r_{1}(m-1)+\sum_{j=2}^{K} T_{1 j} r_{j}(m-1)}{r_{1}(l)+\sum_{j=2}^{K} T_{1 j} r_{j}(l)}<\frac{1}{\varepsilon^{2}} \cdot \frac{\sum_{j=2}^{K} M_{1 j} r_{j}(m-1)}{\sum_{j=2}^{K} M_{1 j} r_{j}(l)} .
$$

Notice that $r_{1}(m-1)=\delta^{k-2} r_{1}(l)$. Suppose

$$
\frac{r_{1}(m-1)}{r_{1}(l)}<\frac{1}{\varepsilon^{2}} \cdot \frac{\sum_{j=2}^{K} M_{1 j} r_{j}(m-1)}{\sum_{j=2}^{K} M_{1 j} r_{j}(l)} .
$$


There must exist some node $j$, such that

$$
r_{j}(m-1)=\delta^{k-2} \cdot \varepsilon^{2} \cdot r_{j}(l)>\delta^{k+1} r_{j}(l)
$$

If not, then from equation (31), it follows

$$
\frac{\sum_{j=2}^{K} T_{1 j} r_{j}(m-1)}{\sum_{j=2}^{K} T_{1 j} r_{j}(l)}<\frac{1}{\varepsilon^{2}} \cdot \frac{\sum_{j=2}^{K} M_{1 j} r_{j}(m-1)}{\sum_{j=2}^{K} M_{1 j} r_{j}(l)} \Rightarrow \frac{\sum_{j=2}^{K} M_{1 j} r_{j}(l)}{\sum_{j=2}^{K} T_{1 j} r_{j}(l)}<\frac{1}{\varepsilon^{2}} \cdot \frac{\sum_{j=2}^{K} M_{1 j} r_{j}(m-1)}{\sum_{j=2}^{K} T_{1 j} r_{j}(m-1)} .
$$

This contradicts the assumption on $\varepsilon$. This concludes the proof to the previous claim.

Since the trajectory is bounded and the states are discrete, the trajectory transits among only a finite set of states and there exists a time instance, $N$, after which the trajectory is periodic. One can show that the period of the trajectory is 1 after $N$. If not, then there is at least one node with a downward run. Let $M$ be the maximum length of all downward runs for all the nodes. The maximum must exist since the number of states is finite. Hence, there exists a node, $j$, and time instances $k$ and $l$, both greater than $N$, such that $r_{j}(k)>\delta^{M+1} r_{j}(l)$. Since $r_{j}(n)$ is periodic for $n>N$, this implies there exists a downward run with length greater than $M$, a contradiction. 
